\title{
Erratum: Mutations in different components of FGF signaling in LADD syndrome
}

Edyta Rohmann, Han G Brunner, Hülya Kayserili, Oya Uyguner, Gudrun Nürnberg, Erin D Lew, Angus Dobbie, Veraragavan P Eswarakumar, Abdullah Uzumcu, Melike Ulubil-Emeroglu, Jules G Leroy, Yun Li, Christian Becker, Kai Lehnerdt, Cor W R J Cremers, Memnune Yüksel-Apak, Peter Nürnberg, Christian Kubisch, Joseph Schlessinger, Hans van Bokhoven \& Bernd Wollnik Nat. Genet. 38, 414-417 (2006).

The name of the 18th author (Christian Kubisch) is spelled correctly here. 